\title{
Classification Method of Aerobics Course Online Teaching Resources Based on Artificial Intelligence Technology
}

\author{
Hua Zhao, ${ }^{1}$ Xin Zhang, ${ }^{1}$ Caixia Wang, ${ }^{2}$ and Weiyu Yuan ${ }^{3}{ }^{3}$ \\ ${ }^{1}$ Department of Sport Arts, Hebei Sport University, Shijiazhuang 050041, Hebei, China \\ ${ }^{2}$ College of Physical Education, Handan University, Handan 056000, Hebei, China \\ ${ }^{3}$ School of Clinical Medicine, Hebei Medical University, Shijiazhuang 050017, Hebei, China \\ Correspondence should be addressed to Weiyu Yuan; 18301113@hebmu.edu.cn
}

Received 19 December 2021; Revised 17 January 2022; Accepted 18 January 2022; Published 9 February 2022

Academic Editor: Xin Ning

Copyright ( $\odot 2022$ Hua Zhao et al. This is an open access article distributed under the Creative Commons Attribution License, which permits unrestricted use, distribution, and reproduction in any medium, provided the original work is properly cited.

In light of the current uneven distribution of aerobics course online resources, this paper presents research on the classification method of aerobics course online teaching resources based on artificial intelligence technology, constructs the display education resource management system based on artificial intelligence technology, realizes the classification of teaching information characteristics, and constructs the classification and evaluation algorithm of aerobiology course online resources. The online teaching resource classification of aerobics course achieves its design goal. Finally, the experiment shows that the artificial intelligence-based online teaching resource classification method for aerobics course is highly practicable and fully meets the research requirements.

\section{Introduction}

Artificial intelligence educational resources have been significantly improved in quantity and quality with the acceleration of educational informatization in China in recent years, serving as a key to the development of educational informatization in China. However, there are some issues, such as large disparities between urban and rural areas and regions, low-level repeated construction, and an information island, to name a few. Sharing Educational Digital Resources Effectively Artificial intelligence technology has become a stumbling block to China's continued scientific development in educational informatization. In the context of the artificial intelligence era, focusing on the development and integration of aerobics teaching resources will not only assist teachers in accepting new knowledge and technology, preparing them to adapt to the new era's curriculum development requirements in teaching practice, and meeting students' demands, thereby improving classroom teaching efficiency, but also improve teachers' and students' innovation and entrepreneurship abilities in the process.

As a result, this paper examines the current situation, existing problems, resource integration strategies, and supporting conditions for the construction of aerobics teaching resources in order to provide useful information for the development of Aerobics Education and teaching, as well as to improve teaching quality and lower education costs by establishing a modern network teaching resource system and introducing more high-quality teaching resources. Simultaneously, we should concentrate on developing an open and adaptable Aerobics Education Resource service platform to enable the integration and sharing of high-quality teaching resources. It is also necessary to fully integrate high-quality teaching resources in order to effectively utilize and plan the internal resources of aerobics, integrate more external resources, comprehensively improve the competitiveness and education and teaching quality of aerobics, and improve the level of talent training. Using the Big Data era as a backdrop, we hope to gather useful data that will aid in improving the quality of aerobics instruction.

\section{Classification of Aerobics Course Online Teaching Resources}

2.1. Construction of Aerobics Course Online Teaching Resource Management System. The online teaching resource management system of aerobics course classifies digital resources from two dimensions: construction status 
and upload status: the first category is the completed and uploaded digital resources; the second category is digital resources that have been built but have not been uploaded [1]. The third category is the digital resources under construction but not uploaded yet. The biggest advantage of this classification method is simple and clear and is easy to integrate digital resources and solve many problems existing in the sharing of digital resources in basic education [2]. The proposed classification model of educational resources includes six aspects: resource application field classification system, resource discipline curriculum classification system, resource applicable object classification system, resource textbook version, resource format classification, and resource type (see Figure 1), so as to classify and locate educational resources.

The object dimension uses the learning object oriented by educational resources as its classification basis, and resources are acted on learners' entities, which aligns with teachers' concerns about resource attributes. Build a hierarchical and object-oriented classification system of applicable objects [3]. Classify the applicable objects to different grades in different educational stages. The classification object's attributes or characteristics determine the classification basis. A variety of different classes are formed by the same or different attributes of information content. A resource type is a category of resource that is defined by the value of one or more attributes. On the one hand, the attributes and main characteristics of artificial intelligence teaching resources should be considered when classifying them [4]. The classification method, on the other hand, should be universal, compatible with existing standards and norms, and extensible enough to allow for the addition of new classifications. This paper summarizes the six classification dimensions shown in Figure 2 after investigating several artificial intelligence learning websites and reviewing relevant literature. In practice, these six dimensions are not only used separately, but also in combination to form a multidimensional classification system that provides richer feature information for resource management, search, and retrieval.

The resource retrieval formula refers to the word search string understood and calculated by the search engine, which is composed of keywords, logical operators, search instructions (search syntax), etc. the keywords are the main body of the retrieval formula [5]. The logical operators and search instructions search the key words from different angles according to the specific query requirements. When simple retrieval and advanced retrieval cannot meet the requirements of visitors, professional retrieval can be used. General visitors do not know much about the writing rules of retrieval, but the resource library will provide help next to professional retrieval to guide visitors how to input retrieval to obtain the required resources [6]. After inputting the compiled search formula in the search area, the visitor can click the "search" button to obtain the search results corresponding to the search formula. The detailed schematic diagram of the resource search module is shown in Figure 3.
The basic education digital resource sharing mechanism is built based on this classification, as shown in the diagram below. Two modules make up the sharing mechanism. The internal dotted box is a mechanism for digital education resources that includes seven internal mechanisms [7]. Outside the internal dashed box, there are four mechanisms that will affect any link in the dashed box. After passing the security monitoring and quality audit mechanisms, highquality digital education resources are added to the standardized sharing platform. All digital education resources are described and classified according to unified standards on this platform, which not only facilitates retrieval, acquisition, and application for users, but also facilitates data sharing and interoperability between different resource management systems, as shown in Figure 4.

The artificial intelligence teaching resources classification system's establishment principle is based on the scientifically sound principle of maintaining a strict hierarchical relationship between teaching resources without compromising their practicability. All teachers and students can benefit from artificial intelligence teaching resources. The system for classifying teaching resources should be straightforward and practical [8]. The purpose of the teaching resource classification system is to ensure that classification topics are integrated, and to use the principle of combining classification retrieval language and topic retrieval language, so that the retrieval language has two query functions: classification retrieval language and topic retrieval language. This is also the direct principle of teaching resource classification. When establishing a classification system, artificial intelligence teaching resources should consider the needs of different users, make artificial intelligence teaching resources serve well, and follow the user-oriented principle [9]. Artificial intelligence teaching resources should have a classification system that meets the needs of information development, with enough space reserved in the classification system for new knowledge to be added to meet people's needs for new knowledge and new disciplines.

2.2. Evaluation Algorithm of Classified Management of Teaching Resources. In order to facilitate learners with similar interests to communicate, share, and recommend learning resources, Shanghai Jiaotong University has developed an agent-based Learning Resource Recommendation Mechanism Based on jade platform [10]. The includes three main panels: "learning resource list," "recommended resource list," and "neighbor list." Click each object in the panel to activate the corresponding function and display it in the right border. "Learning resource list" shows the list of resources that learners have learned. Clicking a resource name can activate the "learned resource details" panel in the right border [11]. After clicking, the detailed content of the resource can be displayed. Learners can read and evaluate the content and recommend the resources they are interested in, as shown in Table 1.

The evaluation index system of artificial intelligence teaching resources promotes the standardization of artificial 


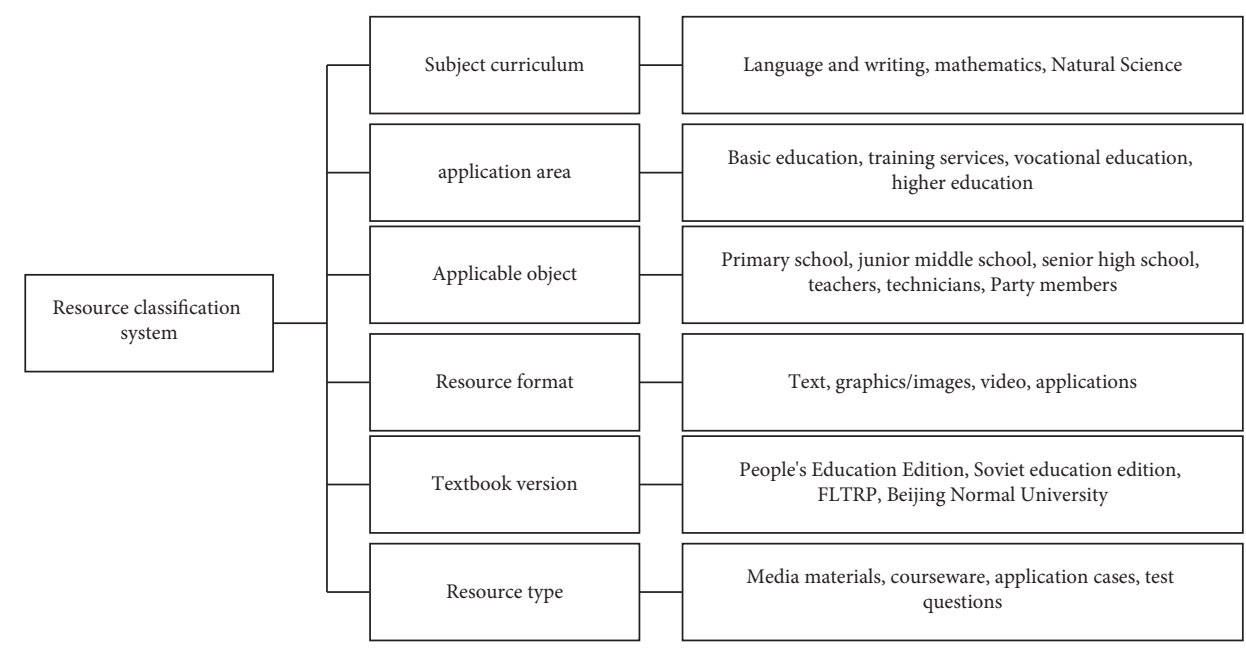

Figure 1: Classification Model of educational resources.

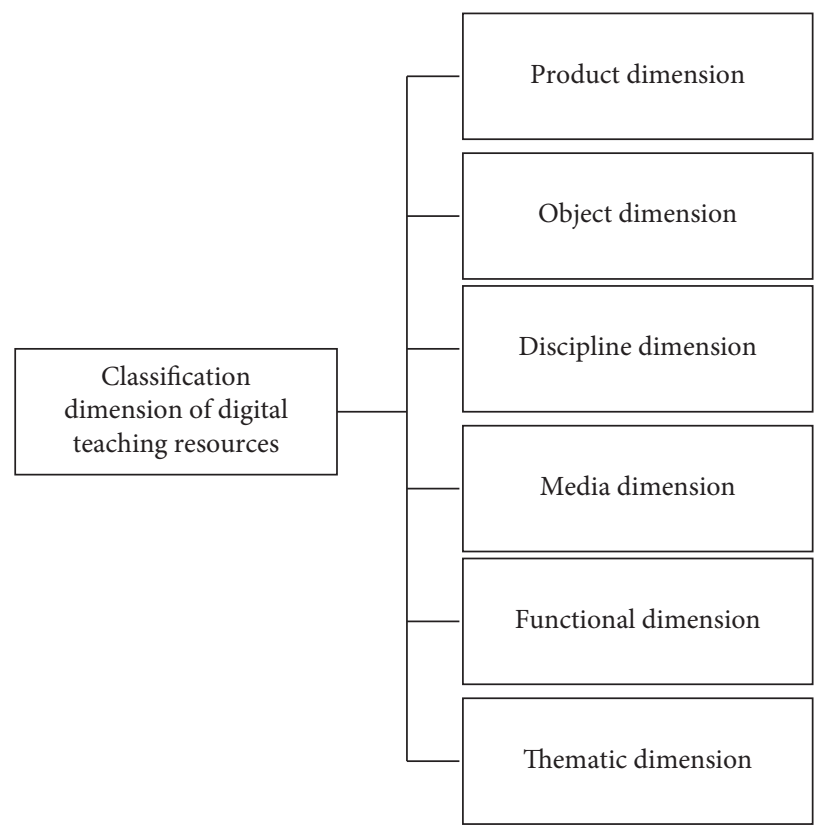

FIGURE 2: Classification dimension of artificial intelligence teaching resources.

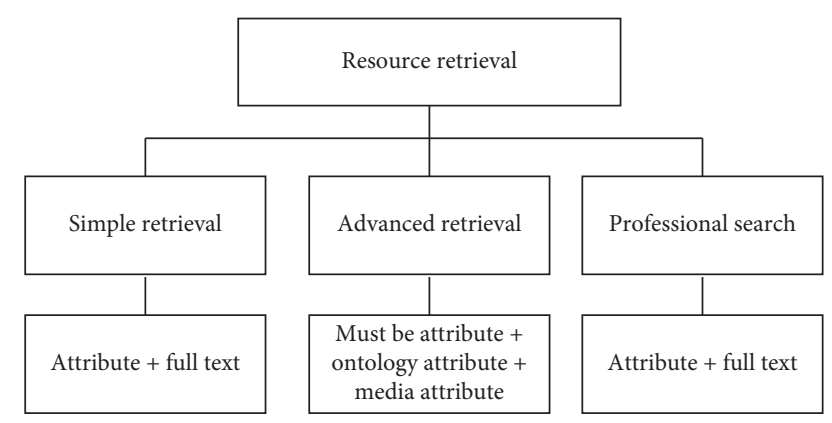

FIGURE 3: Schematic diagram of resource retrieval module. intelligence teaching resources and comprehensively evaluates artificial intelligence teaching resources [12]. The application of artificial intelligence teaching resources in teaching can fundamentally improve teaching methods. In the process of evaluation, the participants take the artificial intelligence teaching resources evaluation index system as the reference basis to help the participants judge the value of teaching resources and determine the evaluation grade $P$. The evaluation work takes the artificial intelligence teaching resources evaluation index system as the measurement standard. When managers input AI teaching resources $T$, they take the evaluation index system as the basis. Excellent AI teaching resources should be reflected through the evaluation index system $f(x)$. People record high-quality AI teaching resources based on the evaluation index system, standardize the construction of AI teaching resources, and improve the quality of AI teaching resource database. The CPU of the physical machine sets the threshold value $\left(L_{i}\right)$ to determine the estimated value $A_{k}^{r}$ of the number of users [13]. If the estimated value is greater than the threshold value, all physical machines will be divided equally to avoid a virtual machine processing too much data at the same time. If the estimated value is less than the threshold value, a PNP cluster subset will be established, and the amount of data $A_{j}$ in it will be equal to the number of physical machines. The specific calculation principle follows the following formula, where a represents the threshold value:

$$
G=\left\{\begin{array}{l}
A_{j}-P t \quad t_{j} \neq 0 \\
\frac{\min }{A_{j}}-\frac{f(x)}{\max } \sum_{i=1}^{n} L_{i} \quad t_{j}=0 \\
A_{k}^{r}+\sum_{i=1}^{n} L_{i} \quad p_{j} \in t_{j} \\
m_{i}(t)=0
\end{array} .\right.
$$




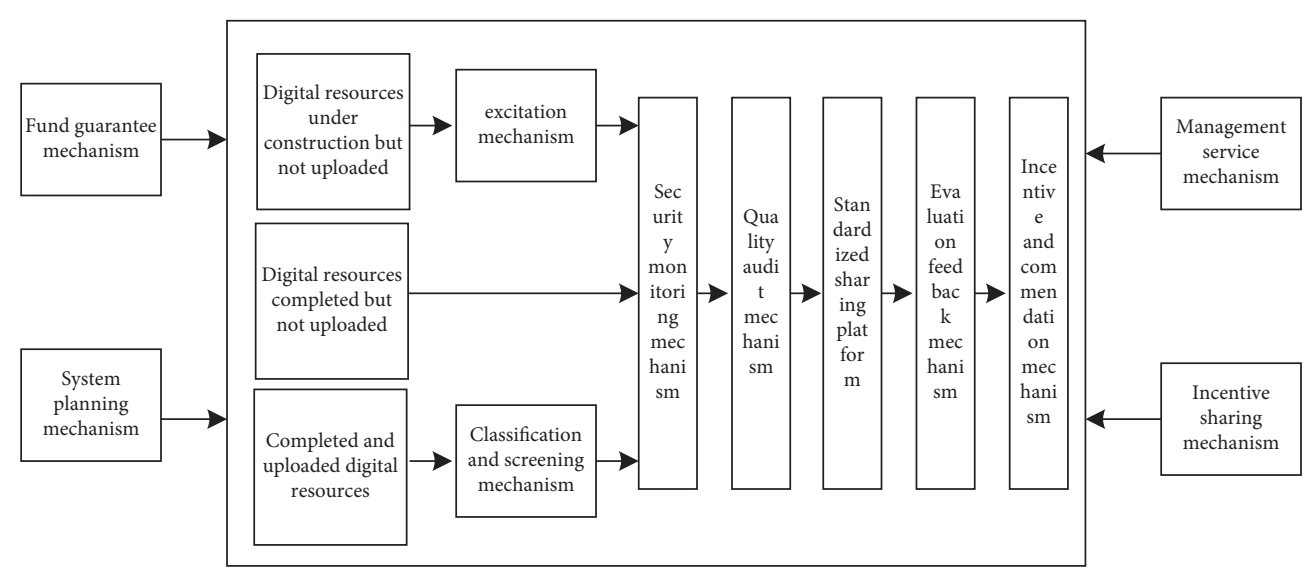

Figure 4: Basic education digital resource sharing mechanism based on resource classification.

TABLE 1: Research status of personalized recommendation mechanism.

\begin{tabular}{lr}
\hline Existing personalized recommendation mechanism & Basic introduction \\
$\begin{array}{l}\text { System } \\
\begin{array}{l}\text { Intelligent recommendation mechanism of self- } \\
\text { evolution in Saskatoon University }\end{array}\end{array} \begin{array}{r}\begin{array}{c}\text { The system mainly uses collaborative filtering technology to generate } \\
\text { recommendation of learning resources according to learners' evaluation } \\
\text { The recommendation mechanism has a wide range of applications. It can actively } \\
\text { analyze the feedback behavior between learners and resources, and obtain learning } \\
\text { resources from the Internet to recommend to learners. }\end{array} \\
\text { ETEC of the University of the Netherlands }\end{array}$ \\
$\begin{array}{r}\text { The purpose of this study is to find potential learning activities and generate } \\
\text { recommendations suitable for learners }\end{array}$ \\
$\begin{array}{r}\text { The system adopts the recommendation mechanism of learning strategies. The } \\
\text { main research still focuses on the construction of conceptual model, and does not } \\
\text { involve practice and verification. }\end{array}$
\end{tabular}

Memory based collaborative filtering technology encompasses both user-based collaborative filtering algorithms and project-based collaborative filtering algorithms. Their most common drawback is that they have sparse data and find it difficult to deal with timely results when dealing with large amounts of data [14]. As a result, developing a modelbased collaborative filtering algorithm is essential. The model-based collaborative filtering algorithm's model training process entails calculating a model using historical data and then using the model to predict [15]. Bayesian network, neural network, latent semantic index, and other technologies are frequently used in model-based collaborative filtering algorithms. We should also consider the algorithm's scalability and complexity when using it in practice.

$$
W=\frac{\sum_{W_{i j}\left|C^{\text {test }}-1\right|}\left|G^{\text {test }}\right|}{A_{j}^{r}-1}-p w .
$$

Among them, $G^{\text {test }}$ is the real score, $W_{i j}$ is the prediction score, and $p w$ is the evaluation diversity of the whole user to be predicted. In order to solve the PRF index defect of information retrieval, a map was invented. The average accuracy of a topic is calculated by calculating the average retrieval accuracy of each relevant document. $R_{i}$ is a single value indicator that reflects the performance of all related documents. The higher the rank of the retrieved related documents, the higher the map. The formula is as follows:

$$
\varpi=\frac{1}{|U-M|} \sum_{i=1}^{|U|} \frac{1}{\left|W-R_{i}\right|}-\frac{1}{r_{i j}}-A B,
$$

where $U$ is the set of test users, $W$ is the number of test user sets, $A$ represents the data of items related to user $B$, and $r_{i j}$ represents the actual preference ranking of user $u$ for the $j$-th related item recommended by user $M$. The recommended set is generated based on the learners' preference for resources, but the learners' preference for resource $\lambda_{u v}$ is expressed as

$$
p(u, s)=\Subset-G \lambda_{u v}+A B-E r_{v s} .
$$

Among them, $r_{v s}$ contains $E$ learners whose interests are closest to those of learner $L, n(s)$ represents the set of learners who have acted on resources, and $\psi$ is the interest similarity between learner $\vartheta$ and learner $\eta$, which represents the user's interest in resources. Since the implicit feedback data of single behavior is used to optimize the teaching resource data control algorithm, the number of mapping structure feature nodes required is $B$, and $A$ is the data distribution node of teaching resources other than our party. Then, the characteristic categories of teaching resource data are divided. The specific algorithm is as follows: 


$$
\begin{aligned}
q_{A B} & =\frac{1}{L}+p(u, s)+P(W-R), \\
\sigma & =R \psi+\frac{q_{A B}}{(M \eta-A B)} \vartheta+\frac{1}{r_{i j}} \\
\theta & =\sigma-m\left(S_{A B}-R_{A B}\right) .
\end{aligned}
$$

In the above expression, $S_{A B}$ is the data control value successfully exchanged between $a$ and $B ; M$ is at a fixed time; $R_{A B}$ is the $m$ value of each time in the process of data exchange, $0<=m<1$; cluster and sort $n$ teaching resource data samples, and divide the nearest cluster $Z$.

$$
d_{i j}=\lim _{0 \longrightarrow \infty} p(u, s)-\frac{\sigma^{m}}{\int^{\infty} \theta}-\frac{1}{\left|W-R_{i}\right|}
$$

The entropy of similar file sets corresponding to the template will decrease, as set similarity increases, according to the formula. The data step is considered the data the user is looking for when the minimum similarity $l \mathrm{~min}$ of teaching resource data is less than the set merging distance. As a result, when establishing the teaching resource data management and control platform, we should fully consider the security of resource and environment mapping and planning, and implement various security prevention technologies and measures to ensure that data is safe, long-term stable, and reliable.

\subsection{Realization of Classification of Aerobics Course Online} Teaching Resources. The main purpose of the resource development guidance specification formulated to cooperate with the artificial intelligence education resource construction project is to unify the behavior of each school in developing network education resources, so that the resources of each school can be shared on a large scale [16]. Its main core is to formulate a series of relevant resource attribute annotation standards according to different resource types. The basic structure is shown in Figure 5.

Home page recommendation, search result recommendation, and behavior intelligent push home page recommendation are three recommended contents in the resource recommendation module. Visitors can choose according to their own needs and arrange them flexibly according to download times, upload times, and user rating if they open the resource library's home page. When a visitor accesses a specific resource after receiving the corresponding retrieval results through the above resource retrieval module, retrieval result recommendation refers to other resources that are highly similar to the resource or belong to the same series. Tourists and logged-in users can both browse the home page and search result recommendations at the same time. The intelligent recommendation of relevant resources to the user based on the user's long-term browsing, downloading, and other operation behaviors in the resource library is known as behavioral intelligence recommendation. It is important to note that the recommendation mode is only available to registered users. The user's long-term operation behavior can only be recorded by logging in to their own account, especially for active users who log in frequently and have a large amount of data to record. The suggested resources are more closely aligned with the requirements. The resource recommendation module's detailed schematic diagram is shown in Figure 6.

According to the relevant standards, there are $11 \mathrm{nec}-$ essary attributes of teaching resources, plus the relevant elements involved in resource evaluation, a total of 16 elements. However, according to the design of this study, "discipline" attribute is "Sports," and "category" is "aerobics." Therefore, only the other necessary attributes are shown in Table 2.

In the complete operation and use process, the teaching resource database generally includes two types of user roles, namely, administrator and user. Administrators have different professional orientations, including administrators and Resource Administrators. The positioning of the teaching resource database designed in this study is to serve teachers and students in junior middle school. Therefore, it is subdivided from the perspective of user identity. As mentioned above, the tourists in the teaching resource database are unregistered pure visitors. Compared with registered users, their use rights are very limited. The operations that can be carried out are only single operations for resources, including resource browsing, retrieval, and download. As mentioned above, the original intention of this paper is that the functional diagram of aerobics teaching resource database is as shown in Figure 7.

The resource library contains a large number of different types of resources. One of the key consideration links of the resource library that wants to have a good user experience is how to enable users to quickly and accurately find the effective resources they need. It is without doubt the goal of multi-dimensional design, and it is the common goal of all resource libraries. The resource retrieval module is the most important module to think about in this paper. Simple retrieval, advanced retrieval, and professional retrieval are three retrieval methods included in the resource retrieval module of the junior middle school Chinese teaching resource database designed in this paper. The visitor enters keywords, words, and sentences in the retrieval box, and the simple retrieval method performs fuzzy retrieval. The retrieval should focus on the resource's most important attributes, such as title and key words. Resource tags, abstracts, and especially text resources are primarily based on these four necessary attributes, with the addition of the attribute description of "image entity" for retrieval for graphics/image resources; "audio prompt" for audio resources; "video prompt" and "subject image" for video resources; and "animation tips" and "content types" for animation resources. Visitors who are unfamiliar with the teaching resource database or who do not require high precision retrieval results should use simple retrieval. By requiring visitors to only enter keyword words and sentences, this retrieval method can filter all resources according to multiple key attributes of resources. Home page recommendation, search result recommendation, and behavior intelligent recommendation are the three recommended 


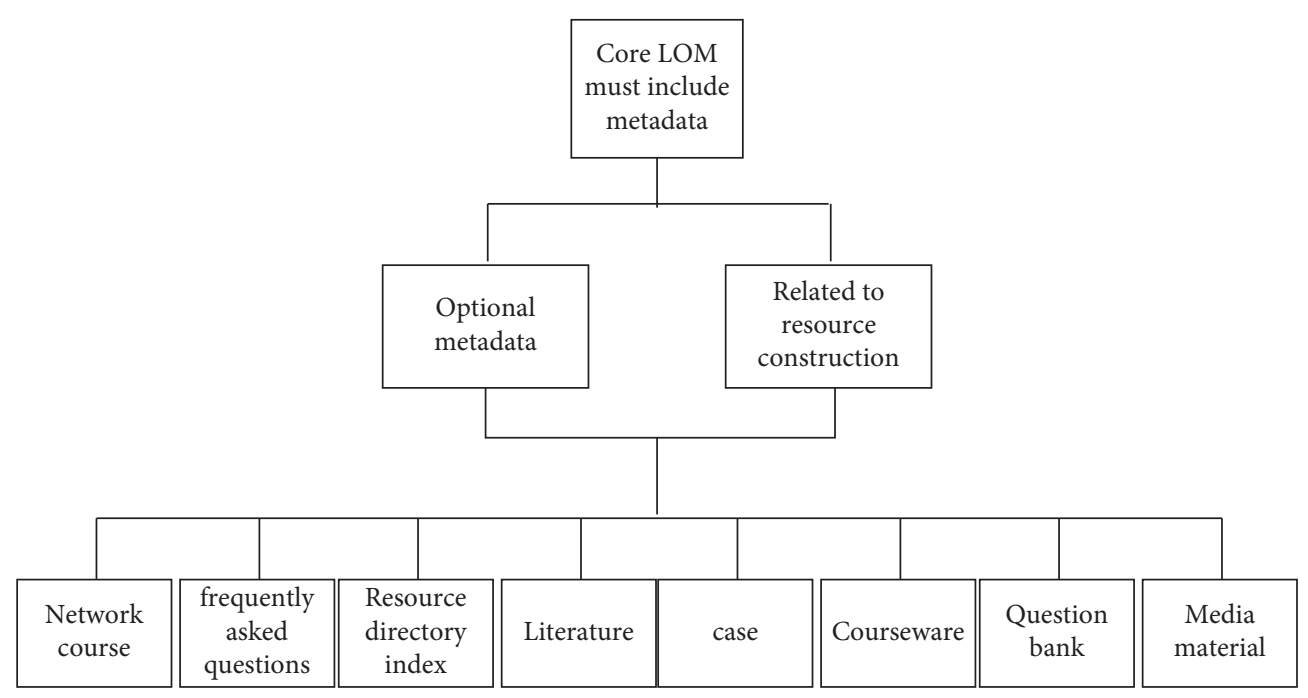

FIgURE 5: Technical specifications for educational resources construction.

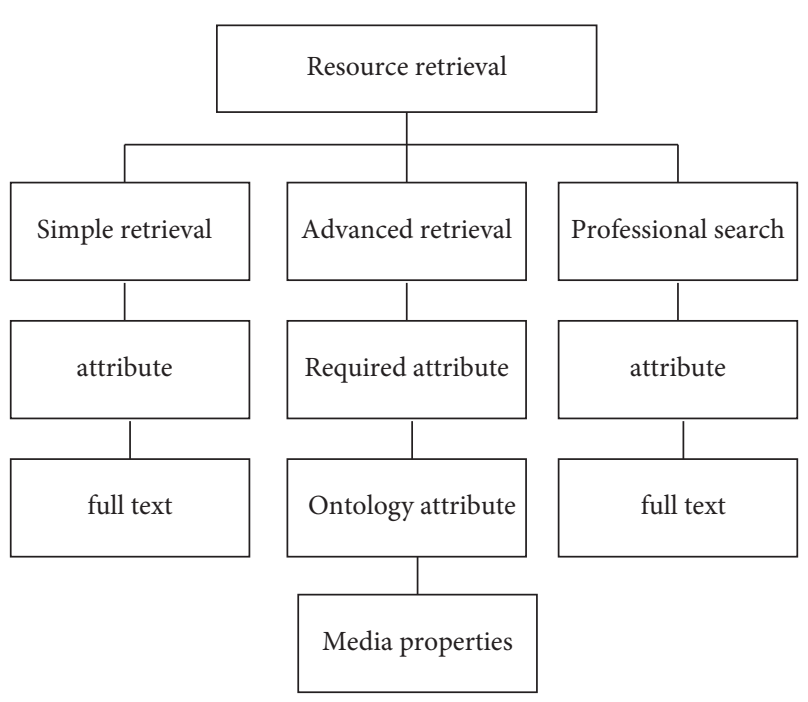

FIGURE 6: Schematic diagram of resource retrieval module.

contents in the resource recommendation module. The resources recommended to visitors when they open the resource library's home page are referred to as "home page recommendations," and visitors can arrange them flexibly according to their own needs based on download times, upload times, and user ratings. When a visitor accesses a specific resource after obtaining the corresponding retrieval results through the above resource retrieval module, retrieval result recommendation refers to other resources that are highly similar to the resource or belong to the same series. Tourists and logged-in users can both browse the home page recommendation and search result recommendation at the same time. The intelligent recommendation of relevant resources to the user based on the user's long-term browsing, downloading, and other operation behaviors in the resource library is referred to as behavioral intelligence recommendation. It is important to note that the recommendation mode is only available to registered users. The user's long-term operation behavior can only be recorded by logging in to their own account, especially for active users who log in frequently, with a large amount of data recorded. The recommended resources are closer to the requirements. The detailed schematic diagram of the resource recommendation module is as shown in Figure 8.

In order to break the isolated island of information resources, better support teaching applications, and realize the sustainability of resource construction, the network architecture of the platform adopts the idea of centralized directory management and distributed storage of resources to form a physically decentralized and logically centralized network system at the provincial, municipal, district, county, and school levels. On the basis of unifying the content construction standards of basic education network resources, comprehensively support the development of teaching activities. The platform model of the network resource center is shown in Figure 9.

The above model is proposed to improve the design according to the problems existing in the current resource database construction in our province. Its bottom layer is a resource library conforming to the metadata specification, including media material library, gauge library, teaching tools and template library, courseware library, case library, literature database, and index directory library. The advantage of conforming to national standards is that it can support the sharing and integration of education and teaching resources in a large area. On the one hand, the specialized search engine helps users quickly retrieve and locate resources; on the other hand, the education and teaching resources scattered on the Internet are continuously supplemented to the library through the data mining and intelligent analysis functions of the search engine. On top of the underlying base library is the special learning resource library for teaching application, which is a dynamic generation library. The resources in the library are created by teachers and students using special learning resources in the teaching process and formed on the basis of processing the contents of the basic library. This can not only meet the personalized needs of teachers' teaching, but also ensure the 
TABLE 2: Required attributes of resources.

\begin{tabular}{lcc}
\hline Name & Explain & Remarks \\
\hline Identification & Label of educational resources & Automatic generation \\
Title & Name of educational resource & Manual completion \\
Key words & Key words describing educational resources & Manual completion \\
Resource tag & A text label describing at least one of the educational resources & Manual completion \\
Abstract & A brief overview of the content of educational resources & Manual completion \\
\hline
\end{tabular}

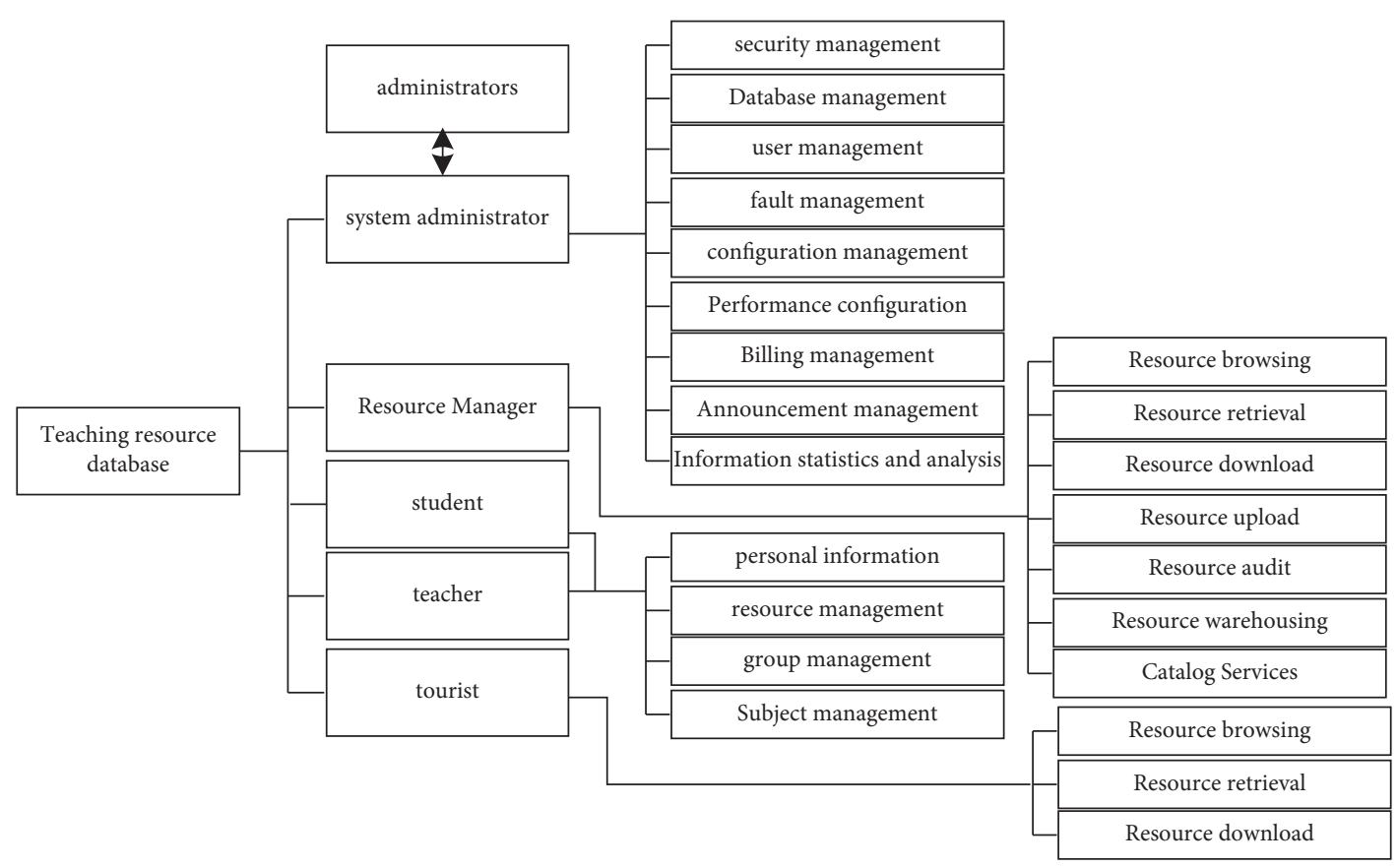

FIGURE 7: Functional structure of teaching resource database.

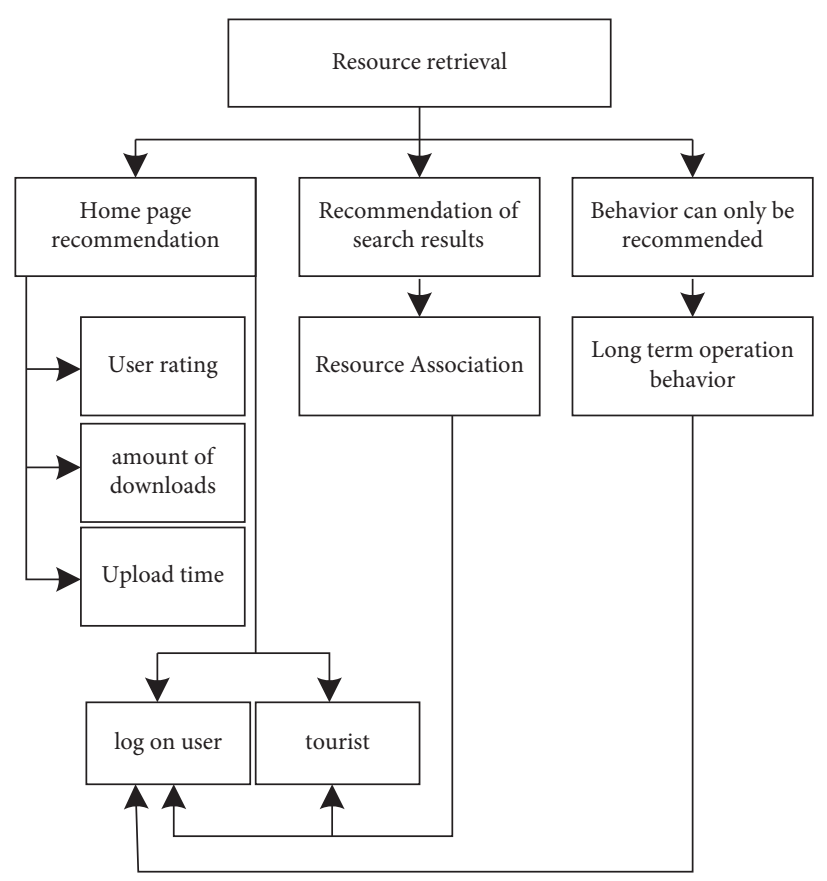

FIGURE 8: Schematic diagram of resource recommendation module. 


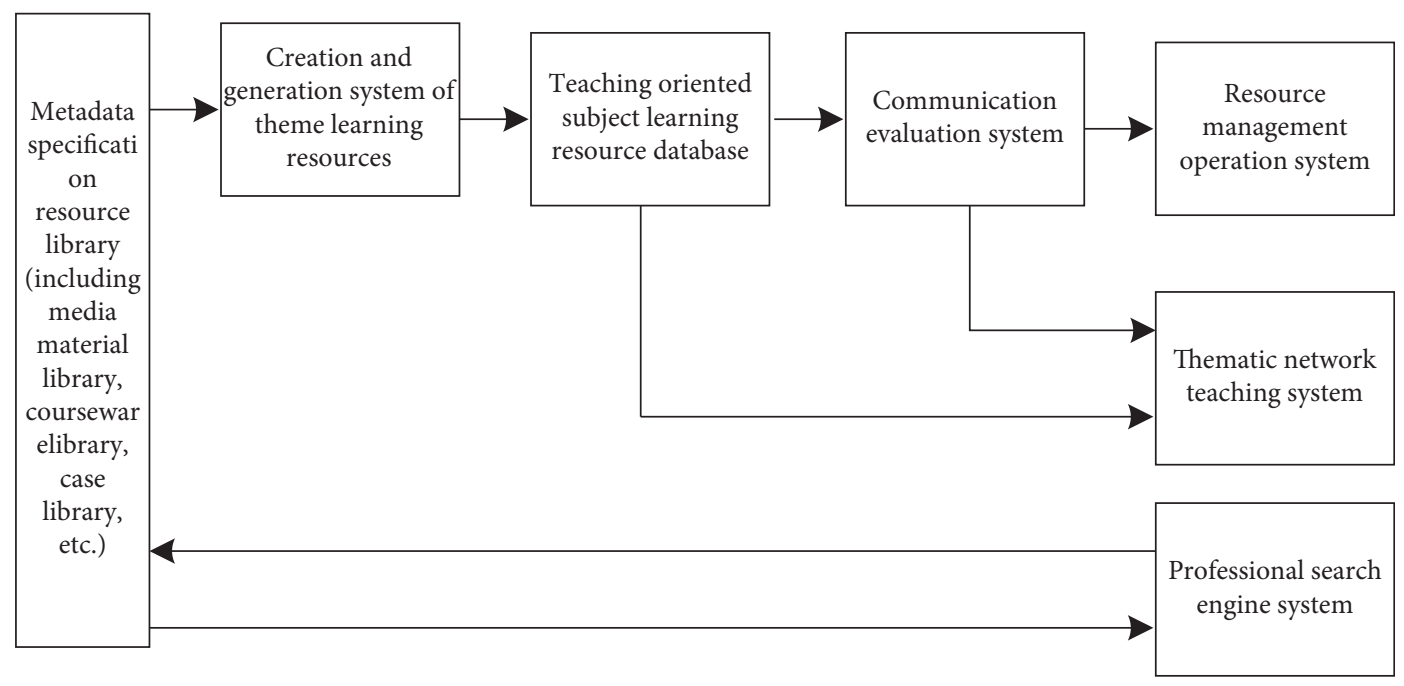

Figure 9: Platform Model of network resource center.

sustainable development of resource database construction, so as to realize the effective classification, storage, and processing of massive teaching resources.

\section{Analysis of Experimental Results}

The aerobics teaching resource database, which is named resource database $\mathrm{A}$, resource database $\mathrm{B}$, and resource database $\mathrm{C}$, is chosen as the evaluation object on the Internet. Then, based on the artificial intelligence teaching resource managers and users' evaluation index table, 20 teaching resource managers and 50 artificial intelligence teaching resource users are invited to score. At the same time, five experts with extensive experience in resource database construction have been invited to assess the benefits and drawbacks of the three aerobics artificial intelligence teaching resources qualitatively. The overall qualitative evaluation of the three aerobics artificial intelligence teaching resources is obtained through the browsing and trial of resource bank $A$, resource bank $B$, and resource bank $\mathrm{C}$ by five experts. Based on a review of the opinions of five experts, it has been determined that resource bank $\mathrm{A}$ has a wide range of resources and a high overall quality level. It is part of a fantastic artificial intelligence teaching resource library. However, resource library C's resources are insufficiently diverse, there are few types, and resource updates are slow. The overall level of quality is a little low. It is part of a library of medium-level artificial intelligence teaching resources. Resource pool B's overall quality is lower than that of resource pools $\mathrm{A}$ and $\mathrm{C}$. The number and types of resources are limited, but those in storage are qualified and useable, making this a largely qualified resource pool. The test is divided into two types of evaluation: artificial intelligence teaching resource managers and artificial intelligence teaching resource users, expressed in quantitative form and divided by score que, by comparing the results of managers and users with the qualitative evaluation results of five experts. A qualified score is $60-69$ points, a medium score is $70-79$ points, a good score is $80-89$ points, and a score of more than 90 points is excellent. The test evaluation results of artificial intelligence teaching resource managers are listed in Table 3.

It can be seen from the table test evaluation results that the scores of the three resource databases are close in terms of indicators, and the scores are very high, indicating that the resource content in the resource database is free of errors and false information, and the reliability is high. Among the secondary indicators, resource pool A has the richest resource content and a wide variety of resources. Many directories in resource pool B are empty and have no actual content. Therefore, resource bank B should strengthen the construction of artificial intelligence teaching resource content and further test the search module of teaching resource sharing service platform, mainly for resource name search and advanced login function, as shown in Tables 4 and 5.

The test of the resource management module of the teaching resource sharing service platform is mainly the test of resource download and evaluation.

All of the functions of the aerobics teaching resource sharing service platform website have passed the above tests. However, we will continue to encounter such issues in the actual use of the aerobics teaching resource sharing service platform management. In order to correct mistakes, we must deal with the research in a timely manner. The effectiveness evaluation criteria are based on automatic classification accuracy and sharing efficiency. The 100 classified resources of the aerobics course artificial intelligence resources are shared using automatic classification sharing and traditional classification sharing. To avoid skewing the results of the comparison, this experiment only uses the period from the start of the search to the end of the sharing operation as the judgment standard. Ten experiments were carried out in order to avoid affecting the comparison results. Figure 10 depicts the results.

As can be seen from Figure 10, when the aerobics course artificial intelligence resources are automatically classified and shared for resource sharing, the resource 
TABLE 3: Trial evaluation results of evaluation indexes of teaching resource database.

\begin{tabular}{|c|c|c|c|c|}
\hline \multirow{2}{*}{ Index } & \multirow{2}{*}{ Fraction } & \multicolumn{3}{|c|}{ Average score of resource pool } \\
\hline & & 1 & 2 & 3 \\
\hline Content & 50 & 46.6 & 36.5 & 38.6 \\
\hline A & 30 & 28.5 & 22.5 & 25.6 \\
\hline B & 20 & 16.5 & 15.8 & 17.8 \\
\hline $\mathrm{C}$ & 20 & 15.2 & 13.5 & 16.5 \\
\hline Form & 22 & 17.5 & 13.5 & 14.8 \\
\hline A & 7 & 5.2 & 4.2 & 4.8 \\
\hline B & 5 & 4.6 & 3.6 & 4.5 \\
\hline $\mathrm{C}$ & 7 & 5.5 & 4.5 & 5.0 \\
\hline $\mathrm{D}$ & 5 & 4.3 & 3.6 & 3.8 \\
\hline Technical & 32 & 25.6 & 20.1 & 25.6 \\
\hline A & 9 & 7.2 & 6.5 & 6.5 \\
\hline B & 9 & 7.1 & 5.2 & 6.9 \\
\hline $\mathrm{C}$ & 8 & 5.6 & 5.3 & 6.5 \\
\hline $\mathrm{D}$ & 8 & 7.5 & 5.5 & 6.2 \\
\hline
\end{tabular}

Table 4: Search effect test.

\begin{tabular}{lccc}
\hline Input & Expected results & Actual results & Pass or not \\
\hline Empty & Search failed & Login failed & Pass \\
JAVA & The search was successful & The search was successful \\
Click the advanced search button & Jump to the advanced search page & Jump to the advanced search page \\
Search by author & The search was successful & The search was successful & Pass \\
\hline
\end{tabular}

TABle 5: Test resource management.

\begin{tabular}{lccc}
\hline Input & Expected results & Actual results & \\
\hline Click the download button & Download start & Download start & Pass or not \\
Resource score & Accept score & Accept score & Yes \\
\hline
\end{tabular}

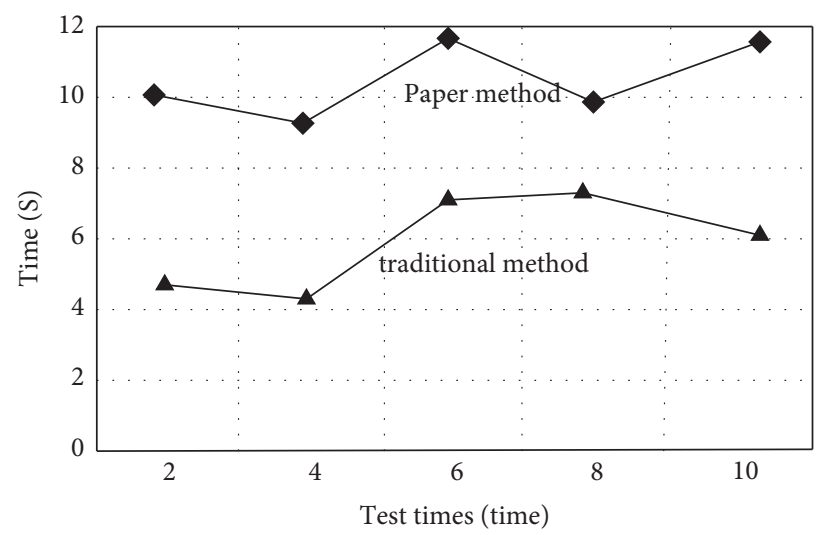

FIGURE 10: Resource sharing efficiency.

query time of the former is shorter than that of the latter. After investigation, it is mainly because the classified resources are automatically classified and shared by the artificial intelligence resources of aerobics courses, which is more convenient to find. Assuming that there are 200 aerobics artificial intelligence teaching resources, they can be divided into five categories in a third-party way. Through the automatic classification and sharing of aerobics artificial intelligence teaching resources, 200 resources are classified, and the results are shown in Table 6.

It can be seen from the table that, based on the automatic classification and sharing of aerobics artificial intelligence teaching resources, the average accuracy is as high as $90 \%$. Based on traditional classification, the average accuracy is only $95 \%$. In contrast, the design accuracy of this paper is higher. 
TABLE 6: Resource classification results of traditional method and this method.

\begin{tabular}{|c|c|c|c|c|c|c|}
\hline & Category & $\mathrm{a}$ & $\mathrm{b}$ & c & $\mathrm{d}$ & $\mathrm{e}$ \\
\hline \multicolumn{2}{|c|}{$\begin{array}{l}\text { Number of resources belonging } \\
\text { to this category/piece }\end{array}$} & 50 & 50 & 50 & 50 & 50 \\
\hline \multirow[t]{3}{*}{ Paper method } & $\begin{array}{c}\text { Number of correctly } \\
\text { classified resources/ } \\
\text { piece }\end{array}$ & 50 & 50 & 50 & 48 & 43 \\
\hline & Accuracy (\%) & 100 & 100 & 100 & 96 & 100 \\
\hline & Average accuracy (\%) & 98 & - & - & - & - \\
\hline \multirow{3}{*}{$\begin{array}{l}\text { Traditional } \\
\text { method }\end{array}$} & $\begin{array}{l}\text { Number of correctly } \\
\text { classified resources/ } \\
\text { piece }\end{array}$ & 47 & 49 & 50 & 47 & 50 \\
\hline & Accuracy (\%) & 90 & 96 & 100 & 92 & 100 \\
\hline & Average accuracy (\%) & 96 & - & - & - & - \\
\hline
\end{tabular}

\section{Conclusion}

Educational resources for various learning objects in various fields have grown significantly in quantity and quality as a result of the rapid development of network education. The demands for precise retrieval, unified management, and sharing of educational resources are becoming increasingly stringent. As a result, scientific descriptions and classifications of educational resources are extremely important. This paper investigates the classification method for aerobics educational resources for artificial intelligence, examines educational resources from six perspectives, application field, subject curriculum, applicable object, resource format, resource type, and textbook version, proposes a village-oriented educational resource model, implements unified coding, and has been successfully implemented in order to provide some guidance for the classification system of educational resources.

\section{Data Availability}

The data used to support the findings of this study are included within the article.

\section{Conflicts of Interest}

All the authors do not have any possible conflicts of interest.

\section{References}

[1] M. Yayla, A. Toma, K.-H. Chen et al., "Nanoparticle classification using frequency domain analysis on resource-limited platforms," Sensors, vol. 19, no. 19, p. 4138, 2019.

[2] B. Krlj, M. Martinc, N. Lavra, and S. Pollak, "autoBOT: evolving neuro-symbolic representations for explainable low resource text classification," Machine Learning, vol. 110, no. 5, pp. 989-1028, 2021.

[3] T. Duan, M. Shaikh, M. Chauhan, J. Chu, and S. Srihari, "Meta learn on constrained transfer learning for low resource cross subject EEG classification," IEEE Access, vol. 8, no. 99, p. 1, 2020.

[4] I. Touahri and A. Mazroui, "Deep analysis of an Arabic sentiment classification system based on lexical resource expansion and custom approaches building," International Journal of Speech Technology, vol. 24, no. 1, pp. 109-126, 2021.
[5] A. Puzanov, S. Zhang, and K. Cohen, "Deep reinforcement one-shot learning for artificially intelligent classification in expert aided systems," Engineering Applications of Artificial Intelligence, vol. 91, pp. 103589.1-103589.12, 2020.

[6] K. Krupcaa and A. Januszewski, "Website and e-shop Development as an e business Teaching Programme Innovation in Management Education," Procedia Computer Science, vol. 176, no. 3, pp. 2476-2486, 2020.

[7] S. Dey, A. Singh, D. Prasad, and K. D. Mcdonald-Maier, "SoCodeCNN: program source code for visual CNN classification using computer vision methodology," IEEE Access, vol. 99, p. 1, 2019.

[8] C. H. Wu, H. S. Lu, and H. M. Hang, "Budgeted passiveaggressive learning for online multiclass classification," IEEE Access, vol. 99, p. 1, 2020.

[9] Z. Zhou, W. Zhou, X. Lv, X. Huang, and H. Li, "Progressive learning of low-precision networks for image classification," IEEE Transactions on Multimedia, vol. 99, p. 1, 2020.

[10] D. Lyons, J. Mack, P. Ketsche, D. Lyons, S. Choo, and M. J. Gallivan, "The impact of inventory leanness and slack resources on supply chain resilience: an empirical study notice to borrowers the impact of inventory leanness and slack resources on supply chain resilience: an empirical study," Human Resource Management Review, vol. 21, no. 3, pp. 243-255, 2019.

[11] Y.Shi, "Image recognition of competitive aerobics movements based on embedded system and digital image processing," Microprocessors and Microsystems, vol. 82, no. 4, Article ID 103925, 2021.

[12] Y. Ma, "Research on the arrangement and visual design of aerobics under the new situation," International Core Journal of Engineering, vol. 5, no. 9, pp. 170-173, 2019.

[13] R. Scherer, S. Howard, J. Tondeur, and F. Siddiq, "Profiling teachers' readiness for online teaching and learning in higher education: who's ready?" Computers in Human Behavior, vol. 118, no. 3, Article ID 106675, 2020.

[14] L. Costa, T. P. Nascimento, and L. Goncalves, "Online learning and teaching of emergent behaviors in multi-robot teams," IEEE Access, vol. 99, p. 1, 2019.

[15] R. Cobos, F. Jurado, and A. Blazquez-Herranz, "A content analysis system that supports sentiment analysis for subjectivity and polarity detection in online courses," Revista Iberoamericana de Tecnologias del Aprendizaje, vol. 99, p. 1, 2019.

[16] L. Ran and Y. He, "Simulation of big data cross access authorization technology based on gradient sampling," Computer simulation, vol. 37, no. 2, p. 5, 2020. 